\title{
We Asked the Experts: Delivering Resilient Surgical Care in a Crisis-Five Survival Strategies for Front-Line Surgeons
}

\author{
Petrut Gogalniceanu ${ }^{1,3} \cdot$ Colin Bicknell ${ }^{2} \cdot$ Tom Reader $^{4} \cdot$ Nick Sevdalis $^{3} \cdot$ Nizam Mamode $^{1,3}$
}

Accepted: 9 August 2020/Published online: 17 August 2020

(C) Société Internationale de Chirurgie 2020

\section{Introduction}

Health care organisations are designed to achieve consistent and reproducible outcomes when faced with planned, predictable or 'routine' emergencies. Unfortunately, the more robust the system, the less agile it is when faced with a novel clinical crisis. This is not surprising, as it is impossible to create emergency operating procedures for every new or unforeseen catastrophe. Similarly, many surgeons in positions of leadership have limited exposure to executive decision-making or clinical expertise outside their area of specialist training. It is not unreasonable therefore for surgical leaders and their organisations to feel overwhelmed by complex and evolving crises, such as the recent COVID-19 pandemic. At such times, it is important to reflect on key strategies that can provide pragmatic, timely and cohesive means of restructuring the delivery of surgical care at an organisational level. We describe five concepts that can help surgical institutions adapt and create a crisis control plan in dynamic circumstances (Table 1).

Petrut Gogalniceanu

petrut.gogalniceanu@gstt.nhs.uk

1 Department of Renal Transplant Surgery, Guy's Hospital, Guy's and St, Thomas' NHS Foundation Trust, 6th Floor, Borough Wing, Great Maze Pond, London SE1 9RT, UK

2 Imperial College London, London, UK

3 King's College London, London, UK

4 London School of Economics, London, UK

\section{Command}

Visible leadership is needed to inspire confidence and instil calm in uncertain times. It is essential therefore that an appropriate ground-level command infrastructure is put into place. Arrangements should also be made for a smooth transition of responsibilities should leaders themselves become incapacitated by illness, the need to isolate or care for family members. In many health care organisations, crisis leadership normally arises from the executive level and is fed down. This model may be inefficient in some crises since it is slow to adapt and respond, is easily overwhelmed with information and can lead to a lack of detailed understanding of local issues. Experience from the Iraq and Afghanistan wars has shown that devolving autonomy to ground commanders is both effective and efficient [1]. As such, health care crises require local clinician leaders that can provide both surgical expertise and executive guidance. Their role is to act as a communication bridge with central leadership, make decisions rooted in the clinical realities of the 'shop floor' and, when needed, use their executive powers to deviate from protocol.

\section{Communications}

Communication is not about what is being said or heard, but about what is understood or perceived [2]. Formal methods of communicating, such as group emails, do not always work well in emergencies. Controversially, mobile communication apps that facilitate group discussions may often provide more agile solutions. Similarly, the use of portable radios may be more appropriate when regular telephony networks fail or when operating in remote hospital areas. More importantly, crisis managers need to 
Table 1 Examples of crisis response strategies applied to clinical practice

\begin{tabular}{|c|c|}
\hline Crisis intervention & Example \\
\hline Command & $\begin{array}{l}\text { Appointment of departmental clinician crisis leaders responsible for specific tasks; e.g. procurement of personal protective } \\
\text { equipment }\end{array}$ \\
\hline Communication & $\begin{array}{l}\text { Use of radios to concomitantly communicate with an entire task group; e.g. a bleeding rapid-response team providing } \\
\text { mobile crisis support in parts of the hospital with poor cell phone signal or across multiple sites } \\
\text { Twice daily team briefings structured according to the NITS framework, enabling team updates and feedback }\end{array}$ \\
\hline $\begin{array}{l}\text { Capacity } \\
\text { management }\end{array}$ & $\begin{array}{l}\text { Development and distribution of action cards to guide non-specialist staff in responding to a specific crisis; e.g. presentation } \\
\text { of a COVID-symptomatic patient to an outpatient clinical area with no acute response capacity }\end{array}$ \\
\hline $\begin{array}{l}\text { Contingency } \\
\text { planning }\end{array}$ & $\begin{array}{l}\text { The use of drill exercises to rehearse team responses to specific crises: e.g. rapidly and safely donning PPE when } \\
\text { responding to a COVID-suspected cardiac arrest }\end{array}$ \\
\hline $\begin{array}{l}\text { Clinical } \\
\text { knowledge }\end{array}$ & $\begin{array}{l}\text { Creation of clinical intelligence teams to inform crisis leaders of evolving internal situations (e.g. depletion of blood } \\
\text { products or oxygen supplies), as well as external events (e.g. the issuing of new national guidance on the management a } \\
\text { clinical condition) }\end{array}$ \\
\hline
\end{tabular}

know how to communicate. Frequent briefing and debriefing cycles can be invaluable in aligning team members' mental models, managing anxieties and collecting feedback from front-line staff. A simple aviation tool for emergency briefing can be used to cover four crisis communication areas [3]: What is the nature and severity of the problem? What are my intentions as a leader? What is the time limit we are working to? What are the special requirements of this situation? The tool is known as NITS $[3]^{1}$ and can be used to structure rapid mini-briefings in crisis situations. These should be followed by opportunities for staff to ask questions and voice concerns. Crucially, the NITS briefing needs to be read back by the recipient(s) to confirm correct perception.

\section{Capacity and resource management}

In crises, all resources are likely to eventually become insufficient, inadequate or inaccessible. Four major types of resource limitations should be addressed:

1. Organisational resources relate to administrative infrastructures, such as policies, procedures or protocols. These need to be rapidly deployed, but also adapted and updated to specific crises. For example, surgical services may need to focus on providing damage control rather than definitive care within an organisation. Furthermore, organisational policies should be ergonomically operationalised-for example using simple action cards or checklists rather than lengthy protocols.

2. Human resources: Staff rostering needs to be sensitive to the realities of emergency situations, such as sick leave amongst the workforce, childcare or inability to access

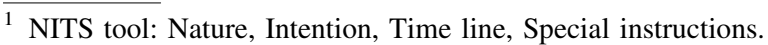

the work place due to a breakdown in transport infrastructure. As such, local crisis managers need to have data on staff availability, skill sets and personal circumstances so that individuals can be redeployed efficiently.

3. Material resources: New tools and technologies may be needed to respond to capacity surges. Surgeons should be particularly aware of the turnaround times for instrument sterilisation and supply of operating room consumables which may become depleted. In addition, investment is needed in infrastructure related to transport, communication and emergency provisions, such as food, water or antibiotics supplies.

4. Physical environments: Crises invariably result in patient overflow. Surgeons can lead the transformation of peri-operative areas into clinical facilities in order to increase surge capacity. Military expertise often provides examples of how to re-purpose or rapidly build physical infrastructures to meet increased demand. Early input from government and military organisations may therefore be needed to address clinical real estate limitations.

\section{Contingency planning}

Contingency planning often relies on the ability of surgical leaders to constantly ask 'what if?' [3]. This allows situational awareness to be maintained and facilitates anticipation of secondary harmful events that may arise further down the line [4]. Drills can be used to stress-test contingency plans [3], being mindful that there is usually a limited timeframe in which these can be rehearsed. It is also important acknowledge that certain crises may not have a solution. This requires the establishment of primary alternatives or backup plans that can be used for damage control purposes. Similarly, bottom lines need to set in 
order to prevent decision-makers from persevering in a futile direction, beyond the limits of safety or common sense.

\section{Clinical knowledge}

Good decision-making needs to be based on clinical evidence or, in its absence, on key principles of surgical practice. Data and expertise are often lacking in the early phases of novel situation and may need to be derived from experts outside the organisation. Surgeons must therefore constantly update their mental models by consulting government and national professional guidance on best clinical practice. Similarly, they need to be sensitive to evolving clinical realities within their own units, such as disease outbreaks in previously 'clean' areas. Data management in a crisis can be facilitated by the creation of separate clinical intelligence teams whose role is to collect, process and disseminate new evidence to clinical leaders.

\section{Summary}

Crisis resilience can be achieved only if surgical institutions remain agile and able to restructure in dynamic circumstances. This requires robust command and communication infrastructures, sagacious resource and data management, as well as contingency planning and rehearsal. Simple interventions, timely deployed and pragmatically implemented are likely to provide solid solutions to unstable situations.

Acknowledgements Dr Mamode reports funding for advisory boards and speakers' expenses from Hansa and Alexion, outside the remit of this study. Dr Sevdalis reports being the director of the London Safety and Training Solutions Ltd, which offers training in patient safety, implementation solutions and human factors to health care organisations. Dr Sevdalis also reports Grants from the National Institute of Health Research (NIHR) Applied Research Collaboration, Grants from King's Improvement Science, Grants from Guy's and St. Thomas' Charity, Grants from the Maudsley Charity, Grants from the ASPIRES research programme, Grants from the NIHR Global Health Research Unit on Health System Strengthening in sub-Saharan Africa, outside the submitted work.

Funding No funding or support were involved in the writing of this article.

\section{Compliance with ethical standards}

Conflict of interest Dr Gogalniceanu, Dr Reader, Dr Bicknell, Dr Mamode and Dr Sevdalis have no related conflicts of interest to declare.

\section{References}

1. McChrystal S (2015) Team of teams-new rules of engagement for a complex world. Penguin, New York

2. Drucker PF (2001) Functioning communication. In: Drucker PF (ed) The essential Drucker. Harper Business, New York

3. Gogalniceanu P (2019) High performance in healthcare-optimising systems, cultures and interventions in surgery [Dissertation]. King's College Library, Department of Abdominal Transplantation, Guy's Hospital, King's College London, London

4. Brennan PA, Holden C, Shaw G, Morris S, Oeppen RS (2020) Leading article: what can we do to improve individual and team situational awareness to benefit patient safety? Br J Oral Maxillofac Surg 58:404-408

Publisher's Note Springer Nature remains neutral with regard to jurisdictional claims in published maps and institutional affiliations. 